\title{
BatchPrimer3: A high throughput web application for PCR and sequencing primer design
}

\author{
Frank M You ${ }^{1,3}$, Naxin Huo ${ }^{1,3}$, Yong Qiang $\mathrm{Gu}^{3}$, Ming-cheng Luo ${ }^{1}$, \\ Yaqin Ma1 ${ }^{1}$ Dave Hane ${ }^{2,3}$, Gerard R Lazo ${ }^{3}$, Jan Dvorak ${ }^{1}$ and \\ Olin D Anderson*3
}

Address: ${ }^{1}$ Department of Plant Sciences, University of California, CA 95616, USA, ${ }^{2}$ Genetic Resources Conservation Program, University of California, CA 95616, USA and ${ }^{3}$ Western Regional Research Center, Agricultural Research Service, US Department of Agriculture, 800 Buchanan Street, Albany, CA 94710, USA

Email: Frank M You - frank.you@ars.usda.gov; Naxin Huo - nhuo@pw.usda.gov; Yong Qiang Gu - yong.gu@ars.usda.gov; Mingcheng Luo-mcluo@ucdavis.edu; Yaqin Ma - ama@ucdavis.edu; Dave Hane-dhane@pw.usda.gov;

Gerard R Lazo - gerard.lazo@ars.pw.usda.gov; Jan Dvorak - jdvorak@ucdavis.edu; Olin D Anderson* - oandersn@pw.usda.gov

* Corresponding author

Published: 29 May 2008

BMC Bioinformatics 2008, 9:253 doi:10.1 I86/147|-2105-9-253

This article is available from: http://www.biomedcentral.com//47I-2105/9/253

(c) 2008 You et al; licensee BioMed Central Ltd.

This is an Open Access article distributed under the terms of the Creative Commons Attribution License (http://creativecommons.org/licenses/by/2.0), which permits unrestricted use, distribution, and reproduction in any medium, provided the original work is properly cited.

\begin{abstract}
Background: Microsatellite (simple sequence repeat - SSR) and single nucleotide polymorphism (SNP) markers are two types of important genetic markers useful in genetic mapping and genotyping. Often, large-scale genomic research projects require high-throughput computer-assisted primer design. Numerous such web-based or standard-alone programs for PCR primer design are available but vary in quality and functionality. In particular, most programs lack batch primer design capability. Such a high-throughput software tool for designing SSR flanking primers and SNP genotyping primers is increasingly demanded.

Results: A new web primer design program, BatchPrimer3, is developed based on Primer3. BatchPrimer3 adopted the Primer 3 core program as a major primer design engine to choose the best primer pairs. A new scorebased primer picking module is incorporated into BatchPrimer3 and used to pick position-restricted primers. BatchPrimer3 v1.0 implements several types of primer designs including generic primers, SSR primers together with SSR detection, and SNP genotyping primers (including single-base extension primers, allele-specific primers, and tetra-primers for tetra-primer ARMS PCR), as well as DNA sequencing primers. DNA sequences in FASTA format can be batch read into the program. The basic information of input sequences, as a reference of parameter setting of primer design, can be obtained by pre-analysis of sequences. The input sequences can be pre-processed and masked to exclude and/or include specific regions, or set targets for different primer design purposes as in Primer3Web and primer3Plus. A tab-delimited or Excel-formatted primer output also greatly facilitates the subsequent primer-ordering process. Thousands of primers, including wheat conserved intron-flanking primers, wheat genome-specific SNP genotyping primers, and Brachypodium SSR flanking primers in several genome projects have been designed using the program and validated in several laboratories.

Conclusion: BatchPrimer3 is a comprehensive web primer design program to develop different types of primers in a high-throughput manner. Additional methods of primer design can be easily integrated into future versions of BatchPrimer3. The program with source code and thousands of PCR and sequencing primers designed for wheat and Brachypodium are accessible at http://wheat.pw.usda.gov/demos/BatchPrimer3/.
\end{abstract}




\section{Background}

Primer design programs are crucial in optimizing the polymerase chain reaction (PCR). A poorly designed primer can result in little or no target product. Numerous web-based or standard-alone programs for PCR primer design are available but vary in quality and functionality $[1,2]$. Primer3 $[3,4]$ is the most popular non-commercial primer design software because of its capabilities and free accessibility. Primer3 core program, a C language-written command line program, has great flexibility to optimize a number of parameters such as product size, melting temperature $\left(T_{m}\right)$, GC content, primer length, 3 ' end stability, self complementarity, primer dimer possibility, position constraints and so forth to get the best primer pairs, and provides the potential to design different types of PCR primers to meet various needs. Due to complexity of Primer3 core program in parameter input, it is difficult to directly use the command line program to design primers. Primer3Web [3] is the first web interface for Primer3 written in Perl. The interface has a powerful, but complex HTML form, including all of possible parameters and options used in the Primer3 core program. Primer3Plus [5] further reorganized and optimized the Primer3Web's user interface in light of parameter categories and primer design tasks. Primer3Web provided two types of primer design, generic primers and hybridization oligos. Primer3Plus further expanded to have cloning and sequencing primer design as well as a primer management module to facilitate further primer analysis and ordering. Several other web-based or command line pipeline programs using Primer3 as a primer design engine also have been developed [6-10]. However, most of those webbased programs lack batch primer design capability. For many large-scale primer design projects, in addition to the requirement of suitable primer design methods, two additional features, batch input of DNA sequences and primer ordering ready output are necessary.

Simple sequence repeat (SSR) and single nucleotide polymorphism (SNP) are two types of important genetic markers. Large numbers of SSRs and SNPs have been detected in various species and used in genetics and breeding [11-15]. A number of different SNP genotyping technologies have been developed based on various methods of allelic discrimination and detection platforms (see review [15]). Primer extension is the most commonly used approach to SNP genotyping because it can be used in a wide variety of high-throughput detection platforms, i.e., electrophoresis, fluorescence resonance energy transfer, fluorescence polarization, arrays, mass spectrometry, and luminescence [15]. A primer extension reaction involves two types of primer design: single base extension primers and allele-specific primers. A software tool for designing SSR flanking primers and SNP genotyping primers in a high-throughput mode is increasingly needed.

On the basis of the Primer3 core program, Primer3Web [3] and Primer3Plus [5], we developed a new web-based application, BatchPrimer3. The aims of BatchPrimer3 development are (1) to implement additional options in primer design, (2) to improve capability of the program to process a large number of DNA sequences, and (3) to provide convenient primer outputs for viewing primer details, printing primer lists, editing primers and finally placing primer orders. We extended Primer 3 Web and Primer3Plus to have batch processing capability of designing primers, and integrated SSR detection and SSR-flanking primer design to have flexible options for SSR search criteria and to export both SSR detection results and SSRflanking primer list. In addition, we implemented primer design methods for two basic types of SNP genotyping primers, single base extension (SBE) primers and allelespecific (AS) primers, as well as tetra-primers for tetraprimer amplification refractory mutation system (ARMS) PCR [16]. DNA sequencing primer design is also reimplemented in this program. The BatchPrimer3 program is easily extendible and additional primer design methods may be integrated in the future.

\section{Implementation \\ Web application design}

BatchPrimer3 was designed as a web application consisting of a set of CGI programs written in Perl, which can run on different operating systems, such as Solaris, Linux, Mac OS or Windows with an Apache HTTP server and Perl interpreter program. The open source program Primer3Web [3] was adopted as a start point. The similar interface in Primer3Plus [5] was used, which has a pulldown combo-box for primer type selection, and a text field together with a button for uploading a sequence file (Figure 1). This task-orientated interface [5] with modular programming design provides extendibility to integrate new primer design methods to the program. File uploading allows users to input a large number of target sequences for batch primer design and overcomes the sequence size limit in an HTML textarea field. The preanalysis module of input sequences is added to calculate sequence properties, such as sequence lengths and GC contents, which is helpful to determine parameter ranges for primer design. The parameter setting panels are customized according to different primer types. When a user chooses a primer type, the corresponding parameter setting panels are represented directly below the sequence input box (Figure 1). An email address text field is also provided to allow a user to receive an email alert of primer design results. 


\section{BatchPrimer3}

\section{a high-throughput web tool for picking PCR and sequencing primers}

BatchPrimer 3 Home I Help I Primer3 Wiki I Copyright Notice and Disclaimer of Primer3 $\mid$ Acknowledgements

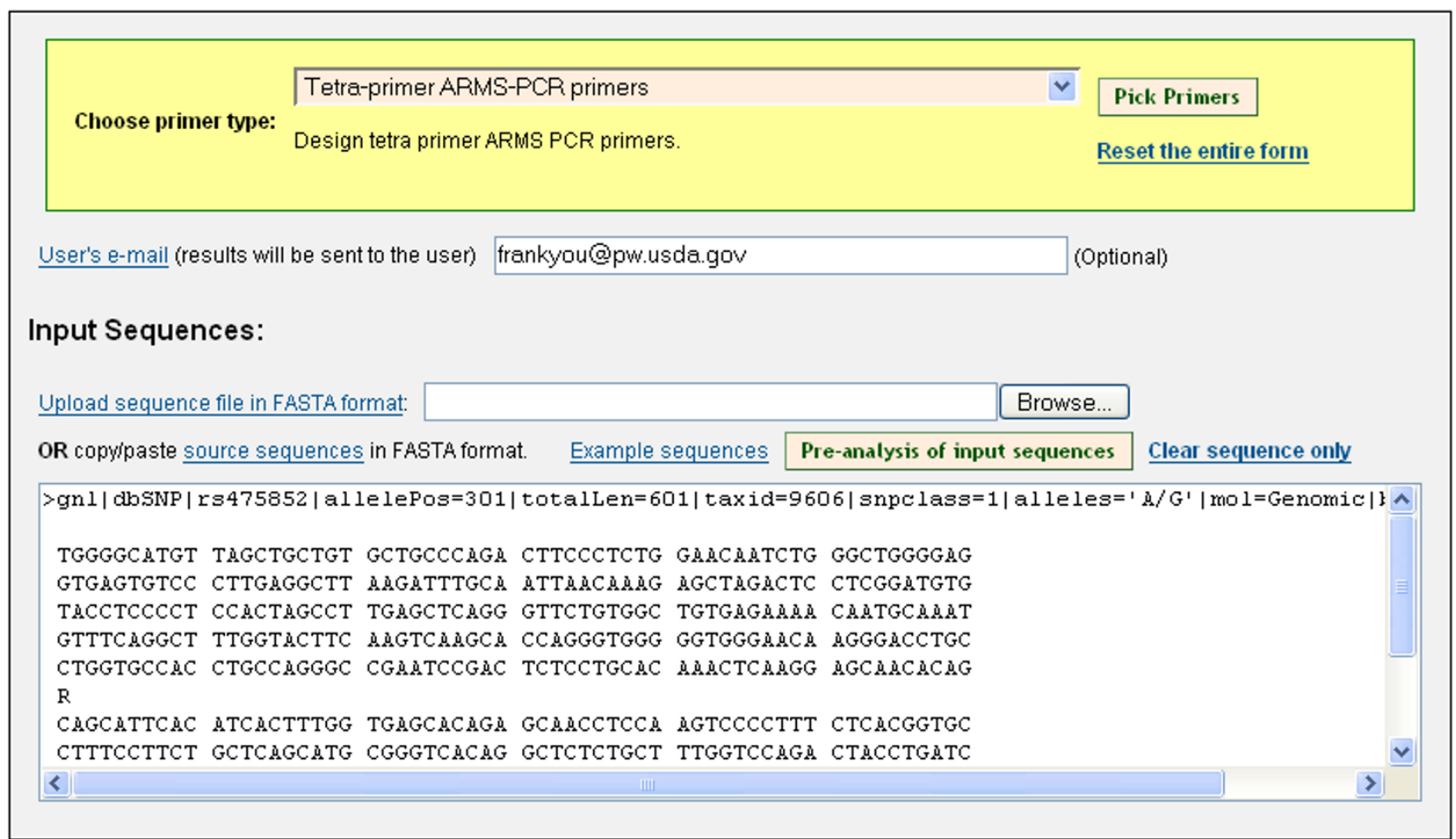

Tetra Primer ARMS PCR Primer Settings

\begin{tabular}{|c|c|c|c|}
\hline Primer Size & Min: 26 & Opt: 28 & $\operatorname{Max}: 30$ \\
\hline Primer Tm & Min: 50 & Opt: 65 & Max: 80 \\
\hline Max Tm Difference: & 5 & & \\
\hline Primer $\mathrm{GC} \%$ & Min: 20 & $\operatorname{Max}: 80$ & \\
\hline$\underline{\text { Inner product size }}$ & Min: 100 & Opt: 150 & $\operatorname{Max}: 200$ \\
\hline$\frac{\text { Relative size difference }}{\text { between inner product sizes }}$ & Min: 1.1 & $\operatorname{Max}: 1.5$ & \\
\hline
\end{tabular}

\section{Figure I}

Web interface of BatchPrimer3 vl.0 application. Various types of primer design can be selected from the primer type pull-up combo-box, and corresponding parameter setting panels are placed below the sequence input box. Pre-analysis of input sequences can be performed before batch primer design.

The Primer3 core program [3] is used to be the major primer design engine for picking the best pairs of standard PCR primers. An additional primer-picking algorithm was implemented to select position-restricted primers such as SBE primers, AS primers and sequencing primers. The best primers are selected based on the quality scores of candi- 
date primers. The quality score is a weighted linear function of primer length, $\mathrm{T}_{\mathrm{m}^{\prime}}$ GC content, number of a singlebase repeat and simple sequence repeats, number of an ambiguity code $(\mathrm{N})$, and self-complementarity of the entire primer and the last 10 nucleotides in the $3^{\prime}$ end. The maximum quality score of a candidate primer is 100 . If the parameter values of a candidate primer are beyond the user-specified ranges, or a candidate primer contains single sequence repeats, the quality score is set to 0 . Within specified parameter range, the closer the user-specified optimum value is to a calculated primer property value, the higher is the primer quality score. If the highest score is zero, no primer is given for the specified criteria. The $T_{m}$ value of a primer varies in different $\mathrm{T}_{\mathrm{m}}$ calculation models - this often results in different set of primers being picked even when using the same $T_{m}$ parameter settings [17]. In BatchPrimer3, the $T_{m}$ of generic primers, hybridization oligo, and SSR flanking primers is calculated using Primer3 core program. An additional $\mathrm{T}_{\mathrm{m}}$ calculation module was implemented for SNP genotyping primers and sequencing primers based on the same model of the nearest neighbour thermodynamic theory $[18,19]$ as used in the Primer3 core program (v 1.1) [4].

\section{Primer design strategy}

Besides generic primer and hybridization oligo [3,5], sequencing primer design is reimplemented in a batch mode. SSR flanking primer design and several SNP genotyping primer designs are newly implemented in BatchPrimer3 v1.0.

\section{SSR screening and primer design}

SSR is a simple repeat of short motifs, 1 to 6 base pairs in length with at least 12 nucleotides in length of SSR [13]. Options of di- to hexa-nucleotide repeat motifs and minimum repeat numbers for each type of motifs are provided in the web interface. An SSR detection algorithm was adopted from the SSR search program [20] to detect the SSR motifs which are then masked as targets. The Primer3 core program is then used to pick the best pairs of primers that flank the targets. If more than one SSR is detected in the same sequence, separate pairs of SSR primers will be designed for each SSR.

\section{Design of primers flanking SNPS}

In most SNP detection platforms, SNP detection requires previous PCR amplification of the genomic region that flanks the SNP site. BatchPrimer3 v1.0 provides a module to design pairs of primers that flank the SNP site.

\section{Design of primers for SNP genotyping}

In BatchPrimer3 v1.0, three types of SNP genotyping primers can be designed: (1) SBE primers, (2) AS primers, and (3) tetra-primers for tetra-primer ARMS PCR system [16].

\section{SBE primer design}

SBE primers are widely used in some high-throughput detection technology platforms, such as SNaPshot (Applied Biosystems) and fluorescence polarization detection (FP-TDI) $[21,22]$. An SBE primer that anneals immediately adjacent to the SNP is extended by one base using a fluorescently labeled ddNTP (Figure 2). For each $\mathrm{SNP}$, it is possible to design two SBE primers, one for each orientation (forward and reverse). For each orientation, all the primer candidates meeting the user-specified primer length range (greater than or equal to the minimum size and less than or equal to the maximum size) are picked. Then the $\mathrm{T}_{\mathrm{m}^{\prime}} \mathrm{GC}$ content and quality score of each candidate are calculated. The primer with the highest score is chosen. A pair of SNP flanking primers and SBE primer can be designed in the same module.

\section{AS primer design}

SNPs can be genotyped using AS primers with the last nucleotide at the $3^{\prime}$ end of a primer corresponding to the site of the SNP [23]. In the AS extension reaction, two primers are required, one for each allele of a SNP (Figure $3)$. AS extension relies on the difference in extension efficiency of DNA polymerase between primers with matched and mismatched 3 ' ends. DNA polymerase extends a primer only when the $3^{\prime}$ end is perfectly complementary to the DNA template. Thus, an AS primer is specific to one of two alleles of a SNP at the 3 ' end of primers and specifically amplifies one of the two alleles. Genotyping is based on determination of the primer that produces the amplicon [15]. If a common reverse primer is used in the reaction, the reaction is called allele-specific PCR (ASPCR) [24-28]. Typically two forward AS primers are used in AS-PCR with a shared reverse non-specific primer. Two PCR reactions are needed to detect both alleles of a SNP $[25,26,28]$. One variant of AS-PCR is to use only one AS
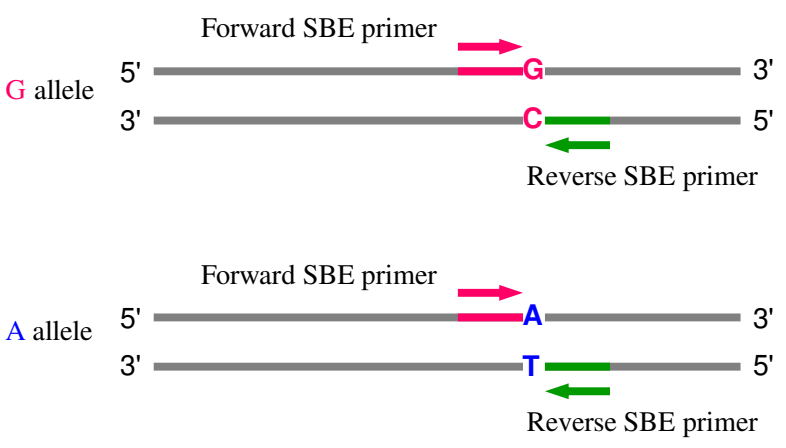

Figure 2

Primer design of single base extension (SBE) primers. One SBE primer is positioned at the base of the $3^{\prime}$ end immediately upstream to the SNP. 
primer and two SNP-flanking primers in one PCR reaction (three-primer nested system) [27]. To enhance the specificity in the AS-PCR reaction, an additional mismatch may be deliberately introduced at the third or other position from the $3^{\prime}$ end of each of the AS primers [16,24-26]. Rules for selection of a nucleotide for the mismatch $[16,24,29]$ are summarized in Table 1: "a 'strong' mismatch $(\mathrm{G} / \mathrm{A}$ or $\mathrm{C} / \mathrm{T})$ at the 3 '-end of an allele-specific primer will likely need a 'weak' second mismatch $(\mathrm{C} / \mathrm{A}$, or $\mathrm{G} / \mathrm{T}$ ) and vice versa, whereas a 'medium' mismatch (A/A, $\mathrm{C} / \mathrm{C}, \mathrm{G} / \mathrm{G}$ or $\mathrm{T} / \mathrm{T}$ ) at the 3 '-end will likely require a 'medium' second mismatch" [16]. An option is provided in the parameter setting panel for adding an additional mismatch and choosing the position of the second deliberate mismatch (the default is the third position). Two sets of AS primers, in both forward and reverse direction can be designed in BatchPrimer3. The SNP-flanking primer pair also can be designed together with AS primers or separately. The same primer selection algorithm is used to choose the AS primers with the highest scores.

\section{Primer design for tetra-primer ARMS PCR}

Ye et al. [16] proposed a simple, effective and economical SNP genotyping method based on AS primers called tetraprimer ARMS-PCR [30-35]. This procedure adopts principles of the tetra-primer PCR method [36] and the amplification refractory mutation system (ARMS) [24]. Four primers are required to amplify a larger fragment from template DNA containing the SNP and two smaller fragments representing each of the two AS products. Primers are designed in such a way that the allelic amplicons differ in size and can be resolved by agarose gel electrophoresis. To enhance the specificity of the reaction, in addition to the first mismatch at the 3' end of AS primers, an extra mismatch is also deliberately introduced at the third position from the 3' end of each of the two inner AS primers (Table 1, Figure 4). From the primer design perspective, two sets of tetra-primers can theoretically be designed for any SNP depending on the AS primer orientation. The schematic diagram of two-set primer design is shown in Figure 4. Although the web program [37] for designing a single set of primers for a SNP is available [16], BatchPrimer3 v1.0 implemented a batch module to easily design two sets of tetra-primers for a SNP.

\section{Program input}

Sequences can be input in two ways. Sequences in FASTA format can be copied and then pasted to the sequence text box (Figure 1). This approach has a maximum size limit of $256 \mathrm{~kb}$. For a large volume of sequences, a FASTA file can be uploaded to the server and the sequence size limitation only depends on Internet speed and server machine memory. When inputting a FASTA file or a single sequence, a header line starting with ">" is mandatory for each sequence. However, empty lines or spaces within sequences are allowed (Figure 1 and 5).

For SNP flanking primer or SNP genotyping primer design, the SNPs or alleles in sequences need to be converted to IUB/IUPAC codes $(\mathrm{G} / \mathrm{C} \rightarrow \mathrm{S}, \mathrm{A} / \mathrm{T} \rightarrow \mathrm{W}, \mathrm{G} / \mathrm{A} \rightarrow \mathrm{R}, \mathrm{T} /$ $\mathrm{C} \rightarrow \mathrm{Y}, \mathrm{G} / \mathrm{T} \rightarrow \mathrm{K}, \mathrm{A} / \mathrm{C} \rightarrow \mathrm{M})$, and the sequence file follows the NCBI dbSNP FASTA format. If multiple SNPs exist in one sequence, BatchPrimer3 will try to design primers for each. Because only one SNP is taken as target and other SNPs are converted to one of the SNP alleles, we suggest that a separate sequence for each SNP is generated based on a reference sequence.

As in Primer3Web [3] and Primer3Plus [5], for any type of primer design, the " \{\} " pair can be inserted into sequences to specify an included region (for example, excluding the vector sequence fragments on both ends), and the " $<>$ " pair to specify excluded regions. An example is to mask all introns with " $<>$ " to design primers only in exons (Figure 5A). An alternative method to specify excluded regions is to replace the unwanted regions with

Table I: The mismatches at the 3' end and the second mismatches at the third or other position from the 3' end in an allele-specific primer

\begin{tabular}{|c|c|c|c|c|}
\hline IUB/IUPAC Code of a SNP & Alleles of a SNP & $\begin{array}{l}\text { Mismatch at the } 3^{\prime} \text { end } \\
\text { (forward/reverse) }\end{array}$ & $\begin{array}{c}\text { Mismatch strength at the } 3^{\prime} \\
\text { end }\end{array}$ & $\begin{array}{l}\text { Second mismatch at the third or } \\
\text { other position from the } 3^{\prime} \text { end }\end{array}$ \\
\hline \multirow[t]{2}{*}{$\mathrm{R}$} & $\mathrm{G} / \mathrm{A}$ & $\mathrm{G} / \mathrm{T}$ & Weak & $\mathrm{G} / \mathrm{A}, \mathrm{T} / \mathrm{C}$ \\
\hline & & $\mathrm{A} / \mathrm{C}$ & Weak & $\mathrm{A} / \mathrm{G}, \mathrm{C} / \mathrm{T}$ \\
\hline \multirow[t]{2}{*}{$Y$} & $\mathrm{~T} / \mathrm{C}$ & $\mathrm{G} / \mathrm{T}$ & Weak & $\mathrm{G} / \mathrm{A}, \mathrm{T} / \mathrm{C}$ \\
\hline & & $\mathrm{A} / \mathrm{C}$ & Weak & $\mathrm{A} / \mathrm{G}, \mathrm{C} / \mathrm{T}$ \\
\hline \multirow[t]{2}{*}{$S$} & $\mathrm{G} / \mathrm{C}$ & G/G & Medium & $\mathrm{C} / \mathrm{C}, \mathrm{A} / \mathrm{A}, \mathrm{G} / \mathrm{G}, \mathrm{T} / \mathrm{T}$ \\
\hline & & $\mathrm{C} / \mathrm{C}$ & Medium & $\mathrm{C} / \mathrm{C}, \mathrm{A} / \mathrm{A}, \mathrm{G} / \mathrm{G}, \mathrm{T} / \mathrm{T}$ \\
\hline \multirow[t]{2}{*}{ W } & $\mathrm{A} / \mathrm{T}$ & $\mathrm{A} / \mathrm{A}$ & Medium & $\mathrm{C} / \mathrm{C}, \mathrm{A} / \mathrm{A}, \mathrm{G} / \mathrm{G}, \mathrm{T} / \mathrm{T}$ \\
\hline & & $T / T$ & Medium & $\mathrm{C} / \mathrm{C}, \mathrm{A} / \mathrm{A}, \mathrm{G} / \mathrm{G}, \mathrm{T} / \mathrm{T}$ \\
\hline \multirow[t]{2}{*}{$\mathrm{K}$} & $\mathrm{G} / \mathrm{T}$ & $\mathrm{G} / \mathrm{A}$ & Strong & $\mathrm{G} / \mathrm{T}, \mathrm{A} / \mathrm{C}$ \\
\hline & & $\mathrm{T} / \mathrm{C}$ & Strong & $\mathrm{T} / \mathrm{G}, \mathrm{C} / \mathrm{A}$ \\
\hline \multirow[t]{2}{*}{$M$} & $\mathrm{~A} / \mathrm{C}$ & $\mathrm{G} / \mathrm{A}$ & Strong & $\mathrm{G} / \mathrm{T}, \mathrm{A} / \mathrm{C}$ \\
\hline & & $\mathrm{T} / \mathrm{C}$ & Strong & $\mathrm{T} / \mathrm{G}, \mathrm{C} / \mathrm{A}$ \\
\hline
\end{tabular}




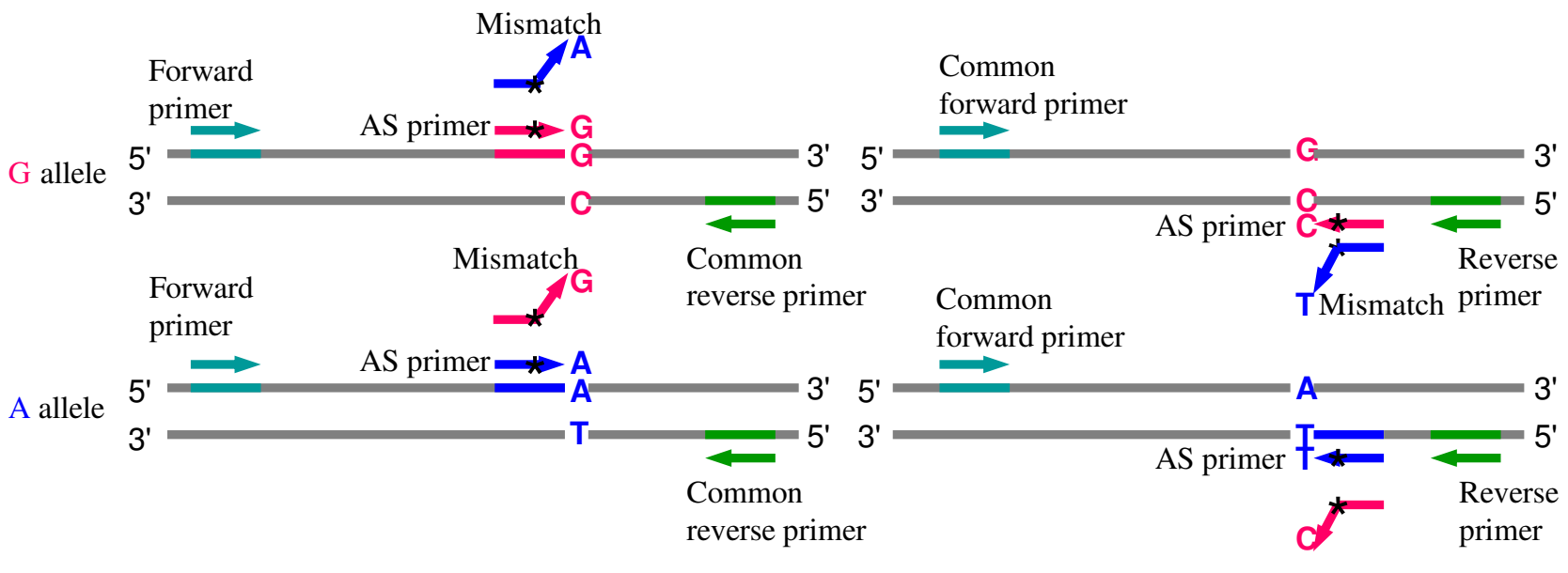

A. Primer set 1

B. Primer set 2

Figure 3

Primer design of allele-specific (AS) primers. Two AS primers, one for each allele of a SNP are designed. The AS primers contain one of two polymorphic nucleotides at the primer 3 ' end. Two sets of primers, either forward or reverse primers can be designed. If a common reverse or forward primer is used in a PCR reaction, the reaction is called allele-specific PCR (AS-PCR). Generally, two PCR reactions are needed for detection of both alleles of a SNP. A variant of AS-PCR is to use only one AS primer and two outer SNP-flanking primers in a single PCR reaction, i.e., three-primer nested system [27]. A mismatch (represented by $*$ ) may be deliberately introduced at the third position from the 3 ' end of each of AS primers to increase allelic specificity (See Table I). The SNP R (G/A) is illustrated as an example, and the other types of SNPs can be applied in the same way.

"N" (Figure 5B) and set the parameter "Max \# Ns" as 0. The " []" pair is adopted to specify targets. If multiple targets are set in one sequence, at least one target will be included in the PCR product [3]. It is notable that target masking can be used only for generic primer design in BatchPrimer3. In addition, multiple targets and excluded regions can be specified in a sequence but only one included region is allowed.

\section{Program output}

The BatchPrimer3 program produces four parts of outputs: (1) a main HTML page containing the primer design summary of all input sequences, (2) an HTML table page listing all designed primers and primer properties, (3) a tab-delimited text file with the same contents in the HTML table page, and (4) a detailed primer view page for each sequence with successfully designed primers (Figure 6). A simple click on the links on the main HTML page or HTML table page will display the primer view. The primer list can be directly saved as a text file or an Excel file for further editing or primer ordering. All primer design results can be downloaded as a zipped file.

\section{Results}

Using the BatchPrimer3 program we have designed thousands of primers in several genomic research projects, including conserved intron-flanking primer pairs from EST sequences for wheat SNP discovery, SNP genotyping primers for wheat SNP mapping, primer pairs from Brachypodium bacteria artificial chromosome (BAC) end sequences for Brachypodium SNP discovery, sequencing primers from EST sequences for gene-specific sequencing, and SSR flanking primer pairs from Brachypodium EST and BAC end sequences for Brachypodium SSR genotyping. Most of these primers have been validated in experiments from several laboratories.

\section{Wheat conserved intron-flanking primer design}

In the project with a goal to discover SNPs in wheat, deletion-mapped wheat EST contigs [38] were compared with rice genomic sequences using BlastN to detect the splice sites (exon/exon junctions) in ESTs. Intron-flanking primers (called conserved primers) were then designed for PCR amplification and sequencing of introns and the nested portions of exons [39]. A total of 6,045 deletion-mapped contigs and singletons were used to perform BlastN searches. Rice introns were inserted into the ESTs at the 


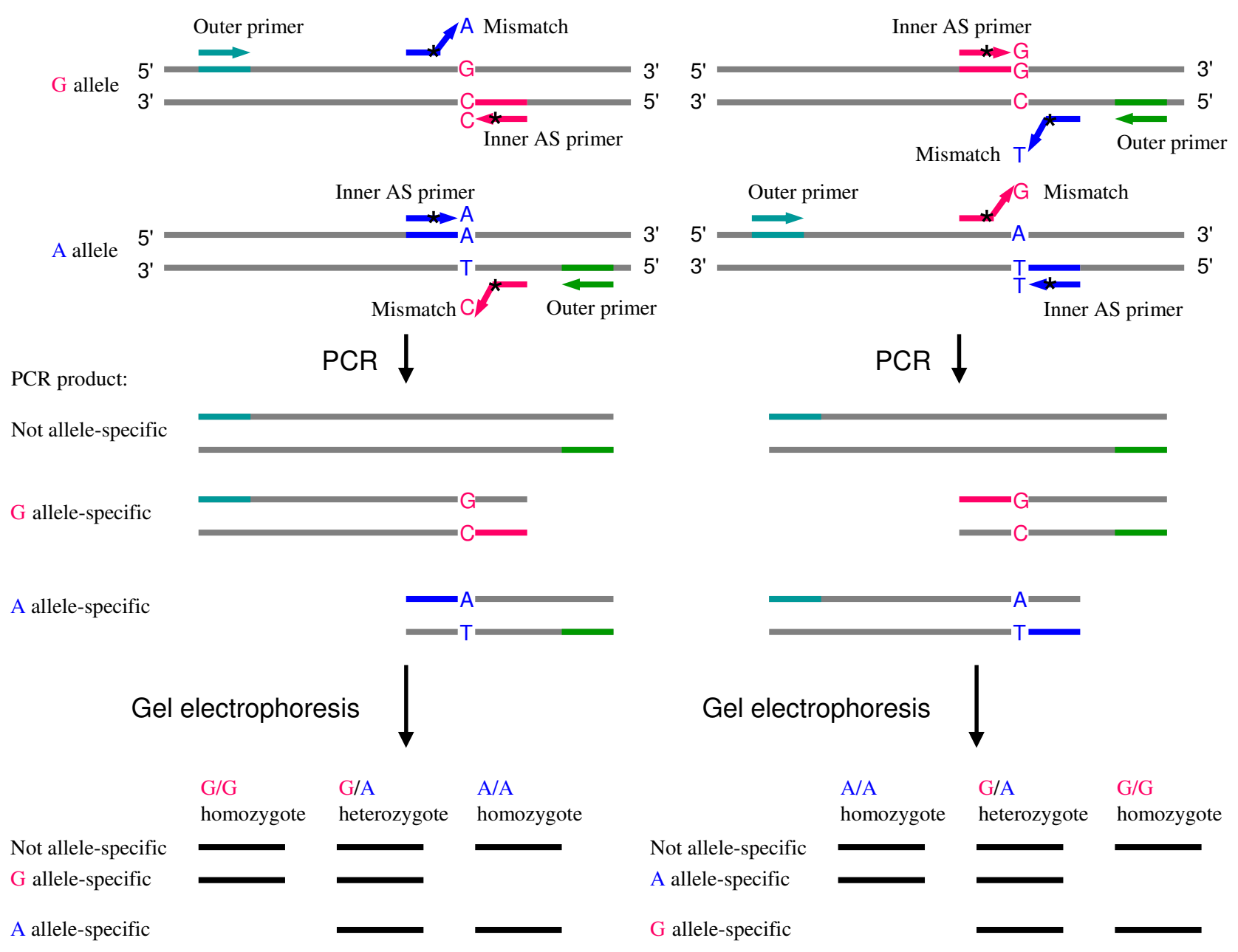

\section{A. Tetra-primer set 1}

B. Tetra-primer set 2

\section{Figure 4}

Schematic illustration of primer design for the tetra-primer ARMS PCR (redraw from Ye et al., 200 I [I6]). The SNP R (G/A) is presented as an example, and the other types of SNPs can be applied in the same way. Four primers, one pair of inner allele-specific (AS) primers and one pair of outer standard primers, are required in a single PCR reaction. Two AS products, one for the $\mathbf{G}$ allele and the other for the $\mathbf{A}$ allele are amplified using two pairs of primers. The former consists of a G AS primer and an outer standard primer, and other latter contains an A AS primer and an outer standard primer. A mismatch (represented by *) is deliberately introduced at the third position from the 3 ' end of each of the two AS primers to increase allelic specificity (See Table I). Two outer standard primers are designed in such a way that the amplicons of two alleles differ in sizes and can be resolved by agarose gel electrophoresis.

predicted positions and replaced with corresponding number of ambiguity codes (Ns) (Figure 5B). PCR primer pairs anchored in neighbouring exons were designed using "Generic primer" module in BatchPrimer3. An additional primer analysis was performed to identify candidate primer pairs that span at least one intron in rice. A total of 2,223 conserved primer pairs were generated. They were further filtered to select only those from single- copy genes. A total of 1,821 of these primer pairs were used for PCR amplification and sequencing of the amplicons from 16 DNAs comprising wheat diploid ancestors and tetraploid wheat in seven different laboratories. Of 240 conserved primer pairs used in one of the laboratories, 228 produced amplicons (95\%). All conserved primers were made publicly available and are downloadable from the wheat SNP web site [40]. 


\begin{abstract}
$>$ BQ162199
TC A AGCTGAGGCAAGCTAAAGACGAAGTAGATAAGCCAGGGCTTCAAGTAA TG<CTTCAAAAGGTGTTGCAACT >ATATGCTTCCAACTTTCTCCGAAAGCGC AGTTACGCTTATAAAGGG<GGAGAGGTTGTAGTGCCTGA>AAAGTTTCTTGA ATCGATAATAGAGGCTCCCGAAAATGACTGGAATAGGCTGTTGCTTGATG GACTTACAGTTGGAAAGGGAGAT<GTTTCACCTGAAGAATTTTA>CGCTGTT ACCAAGAAGAGAATTGAGAGAATCTTGATTCGCACGGAAGGAGGTTCTTA TCAG<CAACGGGTACTTGTCGAATA > TATAAAAGAGATACAAGCTAGAGCAG AgGAAATAGTGAACCGGCTTCAAGGCCCAGCTGTG TAACGTTTATGGTAC AtTtgtAgTTACTGAAAAAgGCTTCGCCCTGCTTtATATATAAAGCACA ATCCACAACAACACGGTACAAACGCACGCCACCAAAAAAAAAAAAAAAAA $\mathrm{AAA}$
\end{abstract}

\begin{abstract}
>BQ162199
TC A AGCTGAGGCAAGCTAAAGACGAAGTAGATAAGCCAGGGCTTCAAGTAA TGNNNNNNNNNNNNNNNNNNNNATATGCTTCCAACTTTCTCCGAAAGCGC AGTTACGCTTATAAAGGGNNNNNNNNNNNNNNNNNNNNAAAGTTTCTTGA ATCGATAATAGAGGCTCCCGAAAATGACTGGAATAGGCTGTTGCTTGATG GACTTACAGTTGGAAAGGGAGATNNNNNNNNNNNNNNNNNNNNCGCTGTT ACCAAGAAGAGAATTGAGAGAATCTTGATTCGCACGGAAGGAGGTTCTTA TCAGNNNNNNNNNNNNNNNNNNNNTATAAAAGAGATACAAGCTAGAGCAG AGGAAATAGTGAACCGGCTTCAAGGCCCAGCTGTG \} TAACGTTTATGGTAC AtTtgtAgTTACTGAAAAAgGCTtTCGCCCTGCTTtATATATAAAGCACA ATCCACAACAACACGGTACAAACGCACGCCACCAAAAAAAAAAAAAAAAA $\mathrm{AAA}$
\end{abstract}

\title{
Figure 5
}

An example of DNA sequence preprocessing. Any unwanted regions for primer design in sequences can be masked using a pair of "<>" to keep the sequence unchanged $(\mathbf{A})$. Alternatively, the unwanted regions can be replaced with "Ns" (B). The included region can be specified by one pair "\{\}" and only one included region can be masked.

\section{Wheat SNP genotyping primer design}

Sequences of amplicons produced with the 1,821 conserved primers were used to design genome-specific primers for PCR amplification of target DNA from a single genome of polyploid wheat and sequencing of the amplicons in a panel of wheat lines and synthetic wheats. A total of 1,527 loci containing one or more SNPs were discovered [14]. The SNPs provide a large number of potential SNP markers. Using this population of SNPs, SBE primers, AS primers, and tetra-primers were designed (Table 2). Theoretically two sets of primers for each SNP can be designed according to primer orientation (Figure 2, 
Sequence ID:

gnl|dbSNP|rs475852|allelePos=301|totalLen=601|taxid=9606|snpclass=1|alleles='A/G'|mol=Genomic|build=126

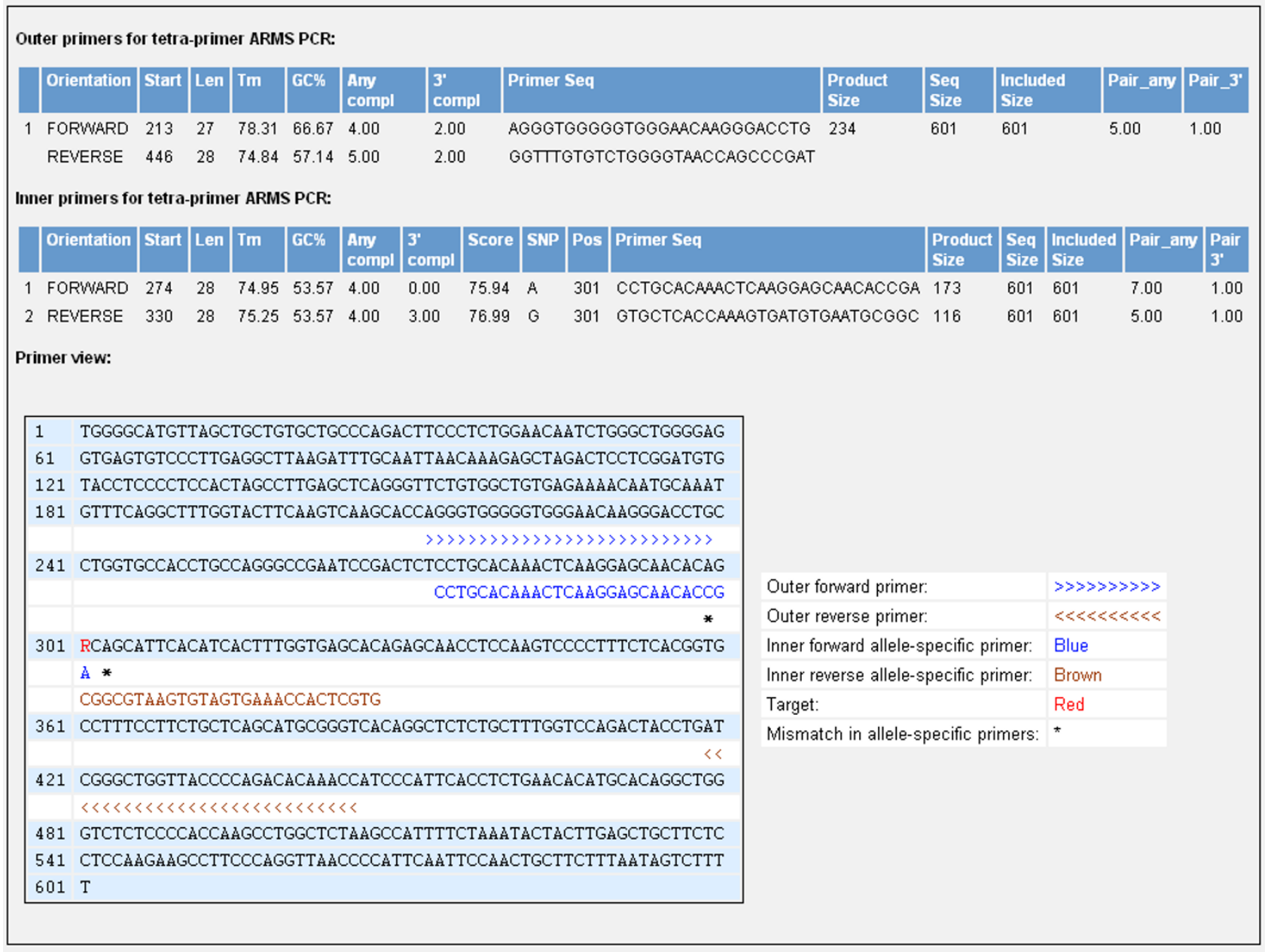

\section{Figure 6}

Screenshot of a primer set in batch primer design. The picture shows the primer design results of sequence ID (rs 1679|736) for tetra-primer ARMS PCR.

3 and 4). Moreover, a gene locus may have several sets of primers for different SNPs or reverse and forward primers. Table 2 lists the number of three types of genotyping primers designed from 1,527 gene loci and their genome and chromosome distribution in wheat. Gene loci rather than sets of primers are reported in Table 2. The primers are derived from 1,186, 1,346, 485 gene loci for the three types of SNP genotyping primers, respectively. The success rates of picking primers based on SNPs was $48.7 \%$, $61.6 \%$, and $12.7 \%$ for the three types of primers, respectively, whereas the success rate based on gene loci was $77.7 \%, 88.2 \%$ and $31.8 \%$, respectively. Because the tetraprimers design requires similar primer properties in two outer primers and two inner AS primers and differing sizes in two inner products, fewer tetra-primers were obtained than of the other two primer types. These primers will be a valuable resource for wheat genetics and breeding. All are accessible at [41]. In addition, 450 SBE primers and corresponding SNP-flanking primer pairs were designed for diploid Aegilops taushii SNPs for their mapping using the SNaPshot assay (Applied Biosystems) (Luo et al., unpublished data). The default parameters for PCR primer design were used, and SBE primers were designed in a range of 25 to 35 bases in primer length, 50 to $90^{\circ} \mathrm{C}$ in $\mathrm{T}_{\mathrm{m}}$ and 20 to $80 \%$ in GC content. Success rate of SBE 
Table 2: The number of wheat SNP genotyping primers designed from I,527 gene loci containing one or more SNPs.

\begin{tabular}{|c|c|c|c|c|c|c|c|c|c|}
\hline \multirow[t]{2}{*}{ Primer type } & \multirow[t]{2}{*}{ Genome } & \multicolumn{7}{|c|}{ Chromosome } & \multirow[t]{2}{*}{ Total } \\
\hline & & I & 2 & 3 & 4 & 5 & 6 & 7 & \\
\hline \multirow[t]{4}{*}{ SBE primer } & A & 51 & 57 & 43 & 63 & 53 & 55 & 54 & 376 \\
\hline & B & 52 & 51 & 47 & 66 & 54 & 53 & 59 & 382 \\
\hline & $\mathrm{D}$ & 58 & 57 & 56 & 56 & 60 & 84 & 57 & 428 \\
\hline & Total & 161 & 165 & 146 & 185 & 167 & 192 & 170 & 1186 \\
\hline \multirow[t]{4}{*}{ AS primer } & A & 62 & 69 & 49 & 69 & 63 & 62 & 62 & 436 \\
\hline & B & 64 & 55 & 54 & 65 & 60 & 65 & 68 & 431 \\
\hline & $\mathrm{D}$ & 67 & 63 & 61 & 67 & 66 & 90 & 64 & 479 \\
\hline & Total & 193 & 187 & 164 & 201 & 189 & 217 & 194 & 1346 \\
\hline \multirow[t]{4}{*}{ Tetra- primer } & $A$ & 17 & 19 & 16 & 25 & 22 & 22 & 20 & $14 \mid$ \\
\hline & B & 20 & 25 & 14 & 19 & 25 & 26 & 27 & 156 \\
\hline & $\mathrm{D}$ & 23 & 23 & 23 & 27 & 30 & 42 & 20 & 188 \\
\hline & Total & 60 & 67 & 53 & 71 & 77 & 90 & 67 & 485 \\
\hline
\end{tabular}

Note: The same parameters were applied to SBE primer and AS primer design: primer length of 25 to 30 bases with the optimum 27 bases, $T_{m}$ of 50 to $65^{\circ} \mathrm{C}$ with the optimum $60^{\circ} \mathrm{C}$. All three types of primers shared the same GC content $(20 \%$ to $80 \%)$, primer complementarity $(\leq 8)$ and $3^{\prime}$ complementarity $(\leq 3)$. Tetra primer design required a primer of 28 bases in length, ranging from 26 to 30 bases, and a broader $\mathrm{T}_{\mathrm{m}}$ range $(50$ to $80^{\circ} \mathrm{C}$ ) with the optimum $65^{\circ} \mathrm{C}$. The optimum inner product size was 120 bases ranging from 100 to 200 bases with a proper size ratio range of two products (from I.I tol.5).

primers in PCR amplification was $82.4 \%$ (371 out of 450 primer sets).

\section{Brachypodium standard primer design}

In the Brachypodium SNP discover project, the non-redundant Brachypodium (accession Bd21) BAC end sequences $[42,43]$ were used to design standard PCR primers for DNA amplification in the accessions Bd21 and Bd3-1, to find SNPs by comparing sequences of the two lines [44]. A total of 960 pairs of primers were designed, 689 $(71.8 \%)$ successfully amplified single products. Approximately one quarter $(28.2 \%)$ of the primers failed to amplify a product in Bd3-1 while producing an amplicon in $\mathrm{Bd} 21$.

\section{Brachypodium SSR detection and SSR-flanking primer design}

To develop SSR markers, the 49,134 Brachypodium BAC end sequences [43] were screened for SSRs and the corresponding SSR-flanking primers were designed. Screening was performed for di-, tri-, tera-, penta- and hexa-nucleotide repeats. The minimum SSR length was set to 12 base pairs and the minimum number of SSR motif repeats were $6,4,3,3,3$, respectively, for di- to hexa-nucleotide repeats. Default parameters for primer design were used: product size of 100 to 300 bases, primer size of 18 to 23 bases with the optimum size of 21 bases, $\mathrm{T}_{\mathrm{m}}$ of 50 to $70^{\circ} \mathrm{C}$ with the optimum at $55^{\circ} \mathrm{C}$ and the maximum difference of $20^{\circ} \mathrm{C}$, and the primer GC content set at 30 to $\% \%$. A total of 10,064 SSRs (1,123 dinucleotide, 3,928 trinucleotide, 3,818 tetranucleotide, 819 pentanucleotide and 376 hexanucleotide) were detected and 8,977 pairs of SSR primers were successfully designed. Genotyping of those SSRs is in progress. The primer list is available at [45].

\section{Performance of BatchPrimer3 web application}

Performance of the BatchPrimer3 program depends on primer type, speed of the server on which BatchPrimer3 resides, client Internet speed (affecting sequence data loading to the server) as well as the efficiency of the BatchPrimer3 and Primer3 core programs. Primer design for generic primer, sequencing primer, and SBE primer performs faster than for other types of primers, with tetraprimer design taking the most time. For example, the above screening of 49,134 sequences for SSR and subsequent primer design took about 526 seconds, whereas the tetra-primer designs from 5,509 sequences took 432 seconds through Internet connections.

\section{Discussion}

\section{Parameter setting}

To obtain high quality primers, primer length, $\mathrm{T}_{\mathrm{m}^{\prime}}, \mathrm{GC}$ content, specificity, and intra- or inter-primer homology must be taken into account [2]. Primer specificity is related to primer length and the final 8 to 10 bases of the 3 ' end sequence. A primer length of 18 to 30 bases is opti- 
mum $[1,2] . T_{m}$ is closely correlated to primer length, GC content and primer base composition. Ideal primer $\mathrm{T}_{\mathrm{m}}$ is in the range of 50 to $65^{\circ} \mathrm{C}$ with GC content in the range of 40 to $60 \%$ for standard primer pairs $[1,2,17]$. However, the optimal primer length varies depending on different types of primers. For example, SNP genotyping primers need a longer primer length ( 25 to 35 bases) to enhance their specificity, and thus the corresponding $\mathrm{T}_{\mathrm{m}}$ might be higher than $65^{\circ} \mathrm{C}$. A suitable $\mathrm{T}_{\mathrm{m}}$ can be obtained by setting a broader GC content range ( 20 to $80 \%$ ). A broader GC content range can increase the success rate of primer picking from sequences with relatively low GC contents (AT rich species or sequences). In BatchPrimer3, the entire primer complementarity and 3' complementarity between and within primers are calculated to assess the intra- or inter-primer homology for the entire primer or 10 bases at the $3^{\prime}$ end. Generally, the score measuring the entire primer complementarity should be less than or equal to 8 and the score for 3' end complementarity should be less than or equal to 3 [3].

\section{Batch primer design}

The advantage of batch primer design is its high efficiency. However, designing primers in the batch mode can result in a failure to design primers for some sequences because input sequences vary in sequence quality and properties (for example, AT rich or GC rich) and/or because the same set of primer design parameters cannot be applied to all sequences. A utility tool for pre-analysis of input sequences is therefore provided in BatchPrimer3 to help users to understand the basic properties of input sequences, such as sequence length and GC content in an entire set of input sequences. The information can be used to adjust the parameter ranges of product size and primer GC content.

The success rate of picking primers in the batch primer design mode is affected by sequence quality, target polymorphism location (for SNP genotyping primers), and parameter settings. Ambiguity codes $(\mathrm{N})$ in a sequence may result in a failure in picking proper primers, especially for position-specific primer design. SBE and AS primers are picked from the region adjacent to, or including the target polymorphism. If an ambiguity code exists in the region or the $\mathrm{T}_{\mathrm{m}^{\prime}}$ GC content or other primer properties cannot meet the parameter settings, primer design will fail. In tetra-primer ARMS PCR, two inner AS products are amplified, which requires that the polymorphism site cannot be too close to an end of a sequence. For SSR primer design, no proper primer is available if an SSR is located at the end of a sequence.

The source of input sequences affects PCR amplification. EST sequences are often used to design SSR primers [11] or other types of primers (such as sequencing primers and conserved primers, see Results). A low amplification rate was reported for EST-derived SSR primers [11] and one of the possible reasons is that one or both primers of the EST-derived SSRs traverse a splice site [11]. The splice site analysis of EST sequences should be performed to mask splice sites or to insert ambiguity codes (Ns) into EST sequences at a splice site (Figure 5B). The wheat conserved primer design strategy is a successful example to resolve this problem.

\section{Conclusion}

BatchPrimer3 is a comprehensive, extendible web primer design program to design different types of PCR and sequencing primers. The batch sequence input and convenient tab-delimited primer outputs facilitate rapid primer design for a large number of sequences and primer ordering. Additional primer design methods can be easily integrated into the program in the future. Using this software program, thousands of primers for wheat and Brachypodium SNP discovery, and SNP and SSR genotyping, have been designed and validated. The program with source code and designed primers can be accessed at [41] (also see Additional file 1).

\section{Availability and requirements Project name: BatchPrimer3.}

Project home page: http://wheat.pw.usda.gov/demos/ BatchPrimer3/.

Operating systems: the software should run in different operating systems, such as Solaris, Linux, Mac-OS or Windows. Tests were performed in Solaris and SuSE Linux systems.

Programming language: Perl

Other requirements: Apache HTTP server, Perl interpreter program

License: GNU PGL

Any restrictions to use by non-academics: None

\section{Authors' contributions}

FMY developed the major modules of the BatchPrimer3 program, designed wheat conserved primers and SNP genotyping primers, and drafted the manuscript. NH and YQG validated wheat conserved primers and Brachypodium primers. M-CL and YM designed and evaluated wheat SBE primers. DH implemented part of the pre-analysis module of input sequences. GRL helped set up the BatchPrimer3 server. JD and ODA participated the design and coordination, and helped to draft the manuscript. All authors read and approved the final manuscript. 


\section{Additional material}

\section{Additional file 1}

BatchPrimer3 application with source code (batchprimer3.tar.gz). This is a tarred and gzipped file, in which there are two directories,

"batchprimer3_cgi-bin" and "batchprimer3_htdocs", and a README.txt file for installation instructions.

Click here for file

[http://www.biomedcentral.com/content/supplementary/14712105-9-253-S1.gz]

\section{Acknowledgements}

This publication is based upon work supported by the U.S. National Science Foundation grant No. DBI-032I757 (the wheat SNP project). We thank P.E. McGuir, the project manager of the project, for his constructive comments in the manuscript. We also thank Primer3 development team for the open source software Primer 3 core program, Primer 3 Web and Primer3Plus.

\section{References}

I. Abd-Elsalam KA: Bioinformatics tools and guideline for PCR primer design. Africa Journal of Biotechnology 2003, 2(5):91-95.

2. Yang $X$, Scheffler BE, Weston LA: Recent developments in primer design for DNA polymorphism and mRNA profiling in higher plants. Plant Methods 2006, 2(I):4.

3. Rozen S, Skaletsky HJ: Primer3 on the WWW for general users and for biologist programmers. In Bioinformatics Methods and Protocols: Methods in Molecular Biology Edited by: Krawetz S, Misener S. Totowa, NJ , Humana Press; 2000:365-386.

4. Koressaar T, Remm M: Enhancements and modifications of primer design program Primer3. Bioinformatics 2007, 23(10):|289-|29|.

5. Untergasser A, Nijveen H, Rao X, Bisseling T, Geurts R, Leunissen JA: Primer3Plus, an enhanced web interface to Primer3. Nucleic Acids Res 2007, 35(Web Server issue):W7I-4.

6. Jewell E, Robinson A, Savage D, Erwin T, Love CG, Lim GA, Li X, Batley J, Spangenberg GC, Edwards D: SSRPrimer and SSR Taxonomy Tree: Biome SSR discovery. Nucleic Acids Res 2006, 34(Web Server issue):W656-9.

7. Hu ZL, Glenn K, Ramos AM, Otieno CJ, Reecy JM, Rothschild MF: Expeditor: a pipeline for designing primers using human gene structure and livestock animal EST information. J Hered 2005, 96(I):80-82.

8. Weckx S, De Rijk P, Van Broeckhoven C, Del-Favero J: SNPbox: a modular software package for large-scale primer design. Bioinformatics 2005, 21 (3):385-387.

9. van Baren MJ, Heutink P: The PCR suite. Bioinformatics 2004, 20(4):59|-593.

10. Raddatz G, Dehio M, Meyer TF, Dehio C: PrimeArray: genomescale primer design for DNA-microarray construction. Bioinformatics 200I, I7(I):98-99.

II. Varshney RK, Graner A, Sorrells ME: Genic microsatellite markers in plants: features and applications. Trends Biotechnol 2005, 23(I):48-55

12. Powell W, Marchray GC, Provan J: Polymorphism revalued by simple sequence repeats. Trends Plant Sci 1996, I:2 I5-222.

13. Temnykh S, DeClerck G, Lukashova A, Lipovich L, Cartinhour S, McCouch S: Computational and experimental analysis of microsatellites in rice (Oryza sativa $L$.): frequency, length variation, transposon associations, and genetic marker potential. Genome Res 200I, I I(8): I44I-I452.

14. Dvorak J, Akhunov ED, Akhunova AR, Anderson OD, Anderson JA, Blake N, Clegg MT, Coleman-Derr D, Conley EJ, Crossman CC, Deal KR, Dubcovsky J, Gill BS, Gu YQ, Hadam J, Heo HY, Huo N, Lazo GR, Lundy KE, Luo MC, Ma YQ, Matthews DE, McGuire PE, Morrell P, Qualset CO, Renfro J, Reynolds S, Dindo T, Talbert LE, Tian C, Toleno D, Warburton M, You FM, Zhang W: Wheat SNP mark- ers: development, mapping and deployment. In Plant \& Animal Genomes XIV San Diego, USA ; 2007.

15. Sobrino B, Brion M, Carracedo A: SNPs in forensic genetics: a review on SNP typing methodologies. Forensic Sci Int 2005, I54:18I-194.

16. Ye S, Dhillon S, Ke X, Collins AR, Day IN: An efficient procedure for genotyping single nucleotide polymorphisms. Nucleic Acids Res 200I, 29(17):E88-8.

17. Dieffenbatch CW, Lowe TMJ, Dveksler GS: General concepts fpr PCR primer design. In PCR primer, A Laboratory Manual Edited by: Dieffenbatch CW, Dveksler GS. New York, Cold Spring Harbor Laboratory Press; 1995: I33-155.

18. Breslauer KJ, Frank R, Blocker H, Marky LA: Predicting DNA duplex stability from the base sequence. Proc Natl Acad Sci USA 1986, 83(I I):3746-3750.

19. SantaLucia J Jr.: A unified view of polymer, dumbbell, and oligonucleotide DNA nearest-neighbor thermodynamics. Proc Natl Acad Sci USA 1998, 95(4): I 460- 1465.

20. SSR finder [ftp://gramene.org/pub/gramene/software/scripts/ssr.pl]

21. Chen X, Levine L, Kwok PY: Fluorescence polarization in homogeneous nucleic acid analysis. Genome Res 1999, 9(5):492-498.

22. Hsu TM, Chen X, Duan S, Miller RD, Kwok PY: Universal SNP genotyping assay with fluorescence polarization detection. Biotechniques 200I, 3 I (3):560, 562, 564-8, passim.

23. Ugozzoli $L$ Wallance, RB: Allele-specific polymerase chain reaction. Methods Enzymol 1991, 2:42-48.

24. Newton CR, Graham A, Heptinstall LE, Powell SJ, Summers C, Kalsheker N, Smith JC, Markham AF: Analysis of any point mutation in DNA. The amplification refractory mutation system (ARMS). Nucleic Acids Res 1989, I 7(7):2503-2516.

25. Drenkard E, Richter BG, Rozen S, Stutius LM, Angell NA, Mindrinos M, Cho RJ, Oefner PJ, Davis RW, Ausubel FM: A simple procedure for the analysis of single nucleotide polymorphisms facilitates map-based cloning in Arabidopsis. Plant Physiol 2000, 124(4): I 483-1492.

26. Zhang W, Gianibelli MC, Ma W, Rampling L, Gale KR: Identification of SNPs and development of allele-specific PCR markers for gamma-gliadin alleles in Triticum aestivum. Theor Appl Genet 2003, 107(I): 130-138.

27. Bundock PC, Cross MJ, Shapter FM, Henry RJ: Robust allele-specific polymerase chain reaction markers developed for single nucleotide polymorphisms in expressed barley sequences. Theor Appl Genet 2006, I I 2(2):358-365.

28. Hayashi K, Yoshida H, Ashikawa I: Development of PCR-based allele-specific and InDel marker sets for nine rice blast resistance genes. Theor Appl Genet 2006, I I3(2):25I-260.

29. Little S: ARMS analysis of point mutations. In Laboratory methods for the Detection of Mutations and Polymorphisms in DNA Edited by: Taylor, G.R.. Boca Raton, FL, CRC Press; 1997:45-5I.

30. Ruiz-Sanz JI, Aurrekoetxea I, Ruiz del Agua A, Ruiz-Larrea MB: Detection of catechol-O-methyltransferase Vall 58 Met polymorphism by a simple one-step tetra-primer amplification refractory mutation system-PCR. Mol Cell Probes 2007, 2I(3):202-207.

31. Leyva-Baca I, Schenkel F, Sharma BS, Jansen GB, Karrow NA: Identification of single nucleotide polymorphisms in the bovine CCL2, IL8, CCR2 and IL8RA genes and their association with health and production in Canadian Holsteins. Anim Genet 2007, 38(3): 198-202.

32. Chiapparino E, Lee D, Donini P: Genotyping single nucleotide polymorphisms in barley by tetra-primer ARMS-PCR. Genome 2004, 47(2):4|4-420.

33. Okayama N, Fujimura K, Nakamura J, Suehiro $Y$, Hamanaka $Y$, Hinoda $Y$ : Evaluation of a new efficient procedure for single-nucleotide polymorphism genotyping: tetra-primer amplification refractory mutation system-polymerase chain reaction. Clin Chem Lab Med 2004, 42(I): $13-16$.

34. Guan F, Yang LG, An JT, Liu SR, Shi GQ: Development of a rapid mismatch PCR method using tetra-primer ARMS for detection of BMPR-IB gene mutation in sheep. Yi Chuan 2005, 27(4):579-583.

35. Piccioli P, Serra M, Gismondi V, Pedemonte S, Loiacono F, Lastraioli S, Bertario L, De Angioletti M, Varesco L, Notaro R: Multiplex tetra-primer amplification refractory mutation system PCR to detect 6 common germline mutations of the MUTYH 
gene associated with polyposis and colorectal cancer. Clin Chem 2006, 52(4):739-743.

36. Ye $S$, Humphries $S$, Green F: Allele specific amplification by tetra-primer PCR. Nucleic Acids Res 1992, 20(5): I I52.

37. Tetra-primer ARMS-PCR [http://cedar.genetics.soton.ac.uk/
[ public html/primer I.html]

38. Qi LL, Echalier B, Chao S, Lazo GR, Butler GE, Anderson OD, Akhunov ED, Dvorak J, Linkiewicz AM, Ratnasiri A, Dubcovsky J, Bermudez-Kandianis CE, Greene RA, Kantety R, La Rota CM, Munkvold JD Sorrells SF, Sorrells ME, Dilbirligi M, Sidhu D, Erayman M, Randhawa HS, Sandhu D, Bondareva SN, Gill KS, Mahmoud AA, Ma XF, Miftahudin, Gustafson JP, Conley El, Nduati V, Gonzalez-Hernandez JL, Anderson JA, Peng JH, Lapitan NL, Hossain KG, Kalavacharla V, Kianian SF, Pathan MS, Zhang DS, Nguyen HT, Choi DW, Fenton RD, Close TJ, McGuire PE, Qualset CO, Gill BS: A chromosome bin map of 16,000 expressed sequence tag loci and distribution of genes among the three genomes of polyploid wheat. Genetics 2004, 168(2):701-7/2.

39. Blake NK, Sherman JD, Dvorak J, Talbert LE: Genome-specific primer sets for starch biosynthesis genes in wheat. Theor Appl Genet 2004, 109(6): 1295-1302.

40. Wheat conserved primers [http://wheat.pw.usda.gov/SNP/new/ pcr primers.shtml]

41. BatchPrimer3 [http://wheat.pw.usda.gov/demos/BatchPrimer3/]

42. Huo N, Gu YQ, Lazo GR, Vogel JP, Coleman-Derr D, Luo MC, Thilmony R, Garvin DF, Anderson OD: Construction and characterization of two BAC libraries from Brachypodium distachyon, a new model for grass genomics. Genome 2006, 49(9): 1099-1108.

43. Huo N, Lazo GR, Vogel JP, You FM, Ma Y, Hayden DM, ColemanDerr D, Hill TA, Dvorak J, Anderson OD, Luo MC, Gu YQ: The nuclear genome of Brachypodium distachyon: analysis of BAC end sequences. Funct Integr Genomics 2008, 8(2): I 35- 147.

44. Garvin DF, Gu YQ, Hasterok R, Hazen SP, Jenkins G, Mockler TC Mur AL, Vogel JP: Development of genetic and genomic research resources for Brachypodium distachyon, a new model system for grass crop research. Crop Sci 2008 48:S69-S84.

45. SSR screening and primers in Brachypodium BAC end sequences [http://brachypodium.pw.usda.gov/SSR/]
Publish with Bio Med Central and every scientist can read your work free of charge

"BioMed Central will be the most significant development for disseminating the results of biomedical research in our lifetime. "

Sir Paul Nurse, Cancer Research UK

Your research papers will be:

- available free of charge to the entire biomedical community

- peer reviewed and published immediately upon acceptance

- cited in PubMed and archived on PubMed Central

- yours - you keep the copyright
BioMedcentral 